\title{
Maximum-Entropy Meshfree Method for Compressible and Near-Incompressible Elasticity
}

\author{
A. Ortiz ${ }^{\mathrm{a}}$, M. A. Puso ${ }^{\mathrm{b}}$, N. Sukumar ${ }^{*, a}$ \\ ${ }^{a}$ Department of Civil and Environmental Engineering, University of California \\ One Shields Avenue, Davis, CA 95616, U.S.A. \\ ${ }^{b}$ Lawrence Livermore National Laboratory, P.O. Box 808, Livermore, CA 94551, U.S.A.
}

Abstract

Numerical integration errors and volumetric locking in the near-incompressible limit are two outstanding issues in Galerkin-based meshfree computations. In this paper, we present a modified Gaussian integration scheme on background cells for meshfree methods that alleviates errors in numerical integration and ensures patch test satisfaction to machine precision. Secondly, a lockingfree small-strain elasticity formulation for meshfree methods is proposed, which draws on developments in assumed strain methods and nodal integration techniques. In this study, maximum-entropy basis functions are used; however, the generality of our approach permits the use of any meshfree approximation. Various benchmark problems in two-dimensional compressible and near-incompressible small strain elasticity are presented to demonstrate the accuracy and optimal convergence in the energy norm of the maximumentropy meshfree formulation.

Key words: linear elasticity, volumetric locking, assumed strain, numerical integration, maximum entropy principle, convex approximation

\footnotetext{
${ }^{*}$ Corresponding author

Email address: nsukumar@ucdavis.edu (N. Sukumar) 


\section{Introduction}

It is well-known that standard displacement-based Galerkin formulations exhibit severe stiffening when modeling near-incompressible materials. In elasticity theory, this occurs when the Poisson's ratio $\nu$ approaches $1 / 2$, and is referred to as volumetric locking. In finite elements, some of the approaches to alleviate locking are: reduced/selective integration [1], $B$-bar technique [2], mixed formulations [3], and assumed strain methods [4]. Displacement-based Galerkin meshfree methods $[5,6,7,8,9,10,11,12,13]$ that are based on moving least squares approximants, natural neighbor interpolants, or entropy approximants are also prone to locking. Huerta and Fernández-Méndez [14] have conducted an indepth study of volumetric locking in the element-free Galerkin (EFG) method. Various remedies have been pursued in the literature to overcome this deficiency - for instance, Dolbow and Belytschko [15] employed reduced integration techniques within a mixed formulation of the EFG method; González et al. [16] enriched the displacement approximation in a mixed natural element formulation; Vidal et al. [17] used pseudodivergence-free approximants in the EFG to satisfy the incompressibility condition; and the $B$-bar and enhanced strain methods were introduced in the EFG by Recio et al. [18]. In an effort to depart from background cell integration, stabilized nodal integration [19, 20, 21, 22, 23] and stress-point integration schemes $[24,25,26]$ have also been proposed to overcome numerical integration errors and facilitate large deformation simulations with meshfree methods. These approaches attempt to mimic reduced integration procedures, and have had success in suppressing volumetric locking. 
Traditionally, numerical integration of the weak form in meshfree methods is carried out using background cells - triangular or quadrilateral elements are typically adopted in two dimensions [6]. Meshfree basis functions are non-polynomial and in addition the support of the basis functions no longer coincides with the union of the background cells that are used in the numerical integration. This leads to inaccuracies in the numerical integration of weak form integrals, and patch test is not passed to machine precision. In the EFG method, Belytschko et al. [6] used higher-order Gauss quadrature in each background cell, and in a subsequent study by Dolbow and Belytschko [27], integration cells that were aligned with the support of the nodal basis functions were used. Griebel and Schweitzer [28] developed a partition of unity meshfree method by formulating a hierarchical algorithm to construct a nodal cover by partitioning the domain into overlapping hyperrectangular patches using $d$-dimensional trees. Due to the overlapping nodal patches, a decomposition of the patches into disjoint cells was performed, and these cells were used as the integration domains. A sparse grid quadrature rule based on univariate Gauss-Patterson rules was employed [29]. As a departure from covers that are rectangular, Riker and Holzer [30] recently proposed a partition-of-unity method in which the nodal cover is a combination of simplexes and polygons.

Atluri et al. [31] proposed a methodology to integrate the weak form in the meshless local Petrov-Galerkin method without the need for background cells by using the support of the basis functions as the domain of integration. This approach was adopted and improved upon in the work of De and Bathe [32]. Similar ideas have also been pursued in Refs. [33, 34, 35, 36]. 
With the aim of using anisotropic weight functions with reduced support sizes, Balachandran et al. [37] developed a methodology that automatically confines the basis functions to natural neighbor polygonal regions by means of the Schwarz-Christoffel mapping. The resulting basis functions are used within a MLS-based meshfree method. Liu and Tu [38] developed an adaptive procedure within individual background cells for meshfree methods. One of the first theoretical studies on the influence of numerical quadrature errors in meshfree methods was recently put forth by Babuška et al. [39]. Schembri et al. [40] compare the performance of different meshfree approximation schemes in three-dimensional computations.

In the past few years, there has been renewed efforts to remedy the poor performance of low-order triangular and tetrahedral finite elements in the near-incompressible regime [41, 42, 43, 44, 45, 46, 47, 48]. These approaches are broadly based on the idea of reducing the number of incompressibility constraints by defining nodal-averaged pressures or strains. In Ref. [48], a special form of a nodal strain matrix was computed from the elements attached to the node, and it led to a locking-free displacement-based formulation. Although these nodal methods tend not to lock, several authors have reported pressure oscillations for highly constrained problems [45, 46].

In this paper, we present new techniques for meshfree methods that provide patch test accuracy in cell-based numerical integration, and a remedy for volumetric locking in the incompressible limit. Our approach for the latter uses the notion of nodal-averaged pressure and leads to a displacementformulation as in Krysl and Zhu [48]; however, we differ in that the starting point of our method is the displacement/pressure mixed formulation, and 
numerical integration is tailored for meshfree basis functions using Gauss quadrature. Since numerical errors are prevalent when standard tensorproduct Gauss quadrature is used in meshfree methods, we appeal to assumed strain methods [49] and nodal integration techniques [20, 21, 22] to define a modified strain tensor. Maximum-entropy basis functions [13, 50] are used to define the modified strain matrix, and Gauss quadrature is adopted in the numerical integration. The procedure so devised alleviates numerical integration errors in meshfree methods using minimal number of integration points and ensures patch test satisfaction to within machine precision.

One of the basic motivations for using meshfree basis functions is they tend to be much more insensitive to poor discretizations and large deformation mesh distortions. Although, automatic mesh generation technology is very mature, there are still instances, particularly in three dimensions, where these mesh generators produce poor tesselations such that near slivers occur. Standard Lagrange shape functions break down in these instances ${ }^{1}$, but meshfree basis functions typically do not. The distortion insensitivity of meshfree methods using integration cells also makes them more robust in large deformations settings. Lagrange elements have a tendency to invert in many tough problems and as such, commercial codes such as LS-DYNA TM offer a meshfree element as an alternative.

The remainder of this paper is structured as follows: In Section 2, a brief introduction to maximum-entropy basis functions is presented. In Section 3, the new formulation for compressible and near-incompressible material be-

\footnotetext{
${ }^{1}$ The element Jacobian, computed from shape function derivatives, becomes singular.
} 
havior is developed. In Section 4, the improved numerical integration scheme is elaborated for two-dimensional background meshes. Numerical examples are presented in Section 5 for benchmark problems in compressible and nearincompressible media to demonstrate the improved numerical integration and the amelioration of volumetric locking. Finally, we close with some final remarks in Section 6.

\section{Maximum-entropy basis functions}

On using the Shannon entropy [51], Jaynes postulated the principle of maximum entropy [52] as a rationale means for least-biased statistical inference when insufficient information is available. The principle of maximum entropy is suitable to find the least-biased probability distribution when there are fewer constraints than unknowns. In the context of meshfree approximants, the probability distribution corresponds to the basis functions $\left\{\phi_{a}\right\}_{a=1}^{n}$ associated with nodes that are located at $\left\{\mathbf{x}_{a}\right\}_{a=1}^{n}$.

The connection between maximum entropy (max-ent) basis functions and linearly complete approximations was established by Sukumar [53]. In Ref. [53], the principle of maximum entropy was employed to obtain linearly complete interpolants on polygonal domains. Arroyo and Ortiz [13] realized a meshfree approximation using a modified entropy functional-with emphasis on establishing a smooth transition between finite element and meshfree methods. Sukumar and Wright [50] generalized the construction of max-ent meshfree basis functions by using the relative (Shannon-Jaynes) entropy functional with a prior [54]. On using compactly-supported prior weight functions that are at least $C^{0}$, compactly-supported max-ent basis 
functions are realized. In particular, when a Gaussian prior is employed the approach of Arroyo and Ortiz [13] is recast. Maximum-entropy basis functions are obtained from a convex optimization problem and are endowed with the following attributes [13]: variation diminishing property; positivedefinite mass matrices and weak Kronecker-delta property on the boundary. The last property is noteworthy since it enables the direct imposition of essential boundary conditions as in finite elements. Recall that most meshfree methods need to resort to special techniques to enforce essential boundary conditions (for example, see Refs. $[55,56,57]$ ). Recently, new applications of max-ent meshfree basis functions have emerged: co-rotational formulation is presented in Ref. [58] and second-order max-ent approximants are proposed in Ref. [59].

We now follow the approach in Ref. [50] to present expressions for max-ent basis functions and their derivatives. To this end, let the prior weight function be denoted by $w_{a}(\mathbf{x})$. The set of max-ent basis functions $\left\{\phi_{a}(\mathbf{x}) \geq 0\right\}_{a=1}^{n}$ is obtained via the solution of the following optimization problem:

$$
\max _{\phi \in \mathbb{R}_{+}^{n}}-\sum_{a=1}^{n} \phi_{a}(\mathbf{x}) \ln \left(\frac{\phi_{a}(\mathbf{x})}{w_{a}(\mathbf{x})}\right),
$$

subject to the linear reproducing conditions:

$$
\sum_{a=1}^{n} \phi_{a}(\mathbf{x})=1, \quad \sum_{a=1}^{n} \phi_{a}(\mathbf{x}) \tilde{\mathbf{x}}_{a}=\mathbf{0}
$$

where we have made use of shifted nodal coordinates $\tilde{\mathbf{x}}_{a}=\mathbf{x}_{a}-\mathbf{x}$. Applying the procedure of Lagrange multipliers, the following expression for max-ent basis functions is obtained [50]:

$$
\phi_{a}(\mathbf{x})=\frac{Z_{a}(\mathbf{x} ; \boldsymbol{\lambda})}{Z(\mathbf{x} ; \boldsymbol{\lambda})}, \quad Z_{a}(\mathbf{x} ; \boldsymbol{\lambda})=w_{a}(\mathbf{x}) \exp \left(-\boldsymbol{\lambda} \cdot \tilde{\mathbf{x}}_{a}\right),
$$


where $Z(\mathbf{x} ; \boldsymbol{\lambda})=\sum_{b} Z_{b}(\mathbf{x} ; \boldsymbol{\lambda})$, and in two dimensions $\tilde{\mathbf{x}}_{a}=\left[\begin{array}{ll}\tilde{x}_{a} & \tilde{y}_{a}\end{array}\right]^{\mathrm{T}}$ and $\boldsymbol{\lambda}=\left[\begin{array}{ll}\lambda_{1} & \lambda_{2}\end{array}\right]^{\mathrm{T}}$. In Eq. (2), the Lagrange multiplier vector $\boldsymbol{\lambda}$ is determined from the dual of the optimization problem posed in Eq. (1) - $\boldsymbol{\lambda}$ is found by minimizing $\ln Z$, which gives rise to the following system of nonlinear equations:

$$
\mathbf{f}(\boldsymbol{\lambda})=\nabla_{\boldsymbol{\lambda}} \ln Z(\boldsymbol{\lambda})=-\sum_{a}^{n} \phi_{a}(\mathbf{x}) \tilde{\mathbf{x}}_{a}=\mathbf{0},
$$

where $\nabla_{\boldsymbol{\lambda}}$ stands for the gradient with respect to $\boldsymbol{\lambda}$. Examples of prior weight functions include Gaussian radial basis functions [13] and quartic polynomials [58]:

$$
\begin{aligned}
& w_{a}(\mathbf{x})=\exp \left(-\beta_{a}\left\|\mathbf{x}_{a}-\mathbf{x}\right\|^{2}\right), \\
& w_{a}(q)=\left\{\begin{array}{lr}
1-6 q^{2}+8 q^{3}-3 q^{4}, & 0 \leq q \leq 1 \\
0, & q>1
\end{array},\right.
\end{aligned}
$$

where $\beta_{a}=\gamma / h_{a}^{2} ; \gamma$ is a parameter that controls the support-width of the basis function at node $a$; and $h_{a}$ is a characteristic nodal spacing that may be distinct for each node $a$. In two dimensions, we set $h_{a}$ as the second-nearest nodal distance to node $a$. For the quartic polynomial, $q=\left\|\mathbf{x}_{a}-\mathbf{x}\right\| / \rho_{a}$ and $\rho_{a}=\gamma h_{a}$ is the radius of the basis function support at node $a$. In the optimization problem, once the converged $\boldsymbol{\lambda}$ is obtained, the basis functions are computed from Eq. (2) and the gradient of the basis function is [58]:

$$
\nabla \phi_{a}=\phi_{a}\left[\tilde{\mathbf{x}}_{a} \cdot\left(\mathbf{H}^{-1}-\mathbf{H}^{-1} \cdot \mathbf{A}\right)+\frac{\nabla w_{a}}{w_{a}}-\sum_{b=1}^{n} \phi_{b} \frac{\nabla w_{b}}{w_{b}}\right]
$$

where

$$
\mathbf{A}=\sum_{b=1}^{n} \phi_{b} \tilde{\mathbf{x}}_{b} \otimes \frac{\nabla w_{b}}{w_{b}}
$$


and $\mathbf{H}$ is the Hessian matrix

$$
\mathbf{H}=\nabla_{\boldsymbol{\lambda}} \mathbf{f}=\nabla_{\boldsymbol{\lambda}} \nabla_{\boldsymbol{\lambda}} \ln Z=\sum_{b=1}^{n} \phi_{b} \tilde{\mathbf{x}}_{b} \otimes \tilde{\mathbf{x}}_{b}
$$

In Fig. 1, plots of a max-ent basis function and its derivatives for a quartic prior function are illustrated. For the Gaussian prior weight function, Eq. (5a) reduces to $[13]$

$$
\nabla \phi_{a}=\phi_{a} \mathbf{H}^{-1} \cdot \tilde{\mathbf{x}}_{a}
$$

\section{Governing equations and mixed formulation}

\subsection{Strong form}

Consider a body defined by an open bounded domain $\Omega \subset \mathbb{R}^{2}$ with boundary $\Gamma$ such that $\Gamma=\Gamma_{u} \cup \Gamma_{t}$ and $\Gamma_{u} \cap \Gamma_{t}=\emptyset$. Linear isotropic elasticity under static loads and no body force with validity for both compressible and nearincompressible material behavior is governed by the following equations [1]:

$$
\begin{aligned}
\boldsymbol{\nabla} \cdot \boldsymbol{\sigma}=0 & \text { in } \Omega, \\
\boldsymbol{\nabla} \cdot \mathbf{u}+\frac{p}{\lambda}=0 & \text { in } \Omega,
\end{aligned}
$$

and the following essential (displacement) and natural (traction) boundary conditions imposed on $\Gamma_{u}$ and $\Gamma_{t}$, respectively:

$$
\begin{array}{r}
\mathbf{u}=\overline{\mathbf{u}} \quad \text { on } \Gamma_{u}, \\
\boldsymbol{\sigma} \cdot \mathbf{n}=\overline{\mathbf{t}} \quad \text { on } \Gamma_{t},
\end{array}
$$




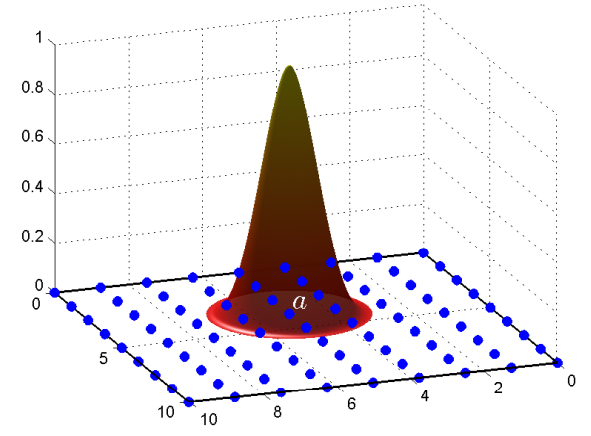

(a)

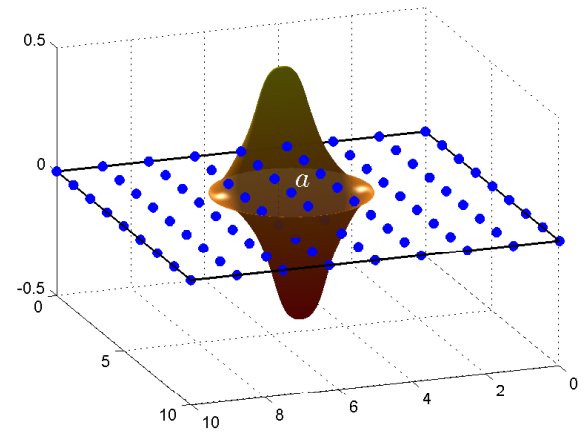

(c)

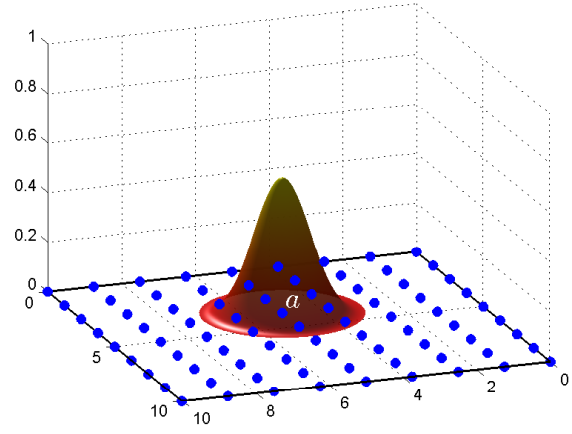

(b)

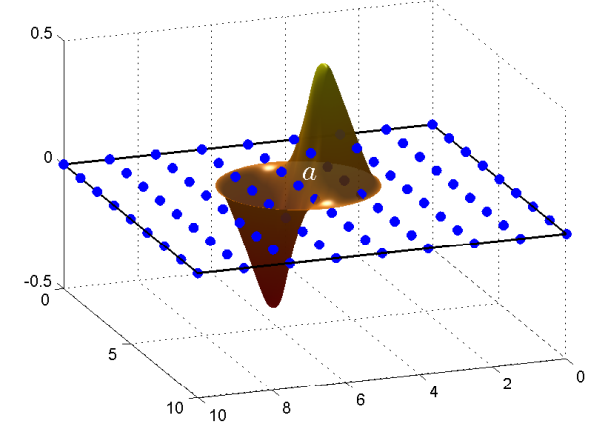

(d)

Fig. 1: Plots of quartic prior weight function and maximum-entropy basis function and its derivatives for node $a$. Note that $w_{a}\left(\mathbf{x}_{a}\right)=1$, but $\phi_{a}\left(\mathbf{x}_{a}\right) \neq 1$, and hence the interior basis function $\phi_{a}$ does not satisfy the Kronecker-delta property. (a) Quartic prior, $w_{a}$; (b) $\phi_{a}$; (c) $\partial \phi_{a} / \partial x$; and (d) $\partial \phi_{a} / \partial y$. 
where the Cauchy stress tensor $\boldsymbol{\sigma}$ is related to the small strain tensor $\boldsymbol{\varepsilon}$ and the pressure parameter $p$ by the following isotropic linear elastic constitutive relation:

$$
\boldsymbol{\sigma}(\mathbf{u}, p)=-p \mathbf{I}+2 \mu \varepsilon(\mathbf{u})
$$

In Eq. (7), $\lambda$ and $\mu$ are Lamé parameters which for plane strain are defined as

$$
\lambda=\frac{\nu E}{(1+\nu)(1-2 \nu)}, \quad \mu=\frac{E}{2(1+\nu)},
$$

where $\nu$ is the Poisson's ratio and $E$ is the Young's modulus of the material. The kinematic relation between the small strain tensor $\varepsilon$ and the displacement vector $\mathbf{u}$ is:

$$
\varepsilon=\frac{1}{2}[\mathbf{u} \otimes \nabla+\nabla \otimes \mathbf{u}]
$$

\subsection{Weak form}

Consider trial functions $u_{i}(\mathbf{x}) \in \mathbb{H}^{1}(\Omega)$ and test functions $\delta u_{i}(\mathbf{x}) \in \mathbb{H}_{0}^{1}(\Omega)$ $(i=1,2)$, where $\mathbb{H}^{1}(\Omega)$ is the Sobolev space of functions with squareintegrable first derivatives in $\Omega$, and $\mathbb{H}_{0}^{1}(\Omega)$ is the Sobolev space of functions with square-integrable first derivatives in $\Omega$ and vanishing values on the essential boundary $\Gamma_{u}$. Also, the trial function for the pressure parameter $p \in \mathbb{H}^{0}(\Omega)$ and test functions $\delta p \in \mathbb{H}^{0}(\Omega)$, where $\mathbb{H}^{0}(\Omega) \equiv L^{2}(\Omega)$ is the Sobolev space of square-integrable functions. The weak form (principle virtual work) for the displacement/pressure mixed formulation is [1]:

$$
\begin{array}{rlrl}
\int_{\Omega} \delta \varepsilon_{i j} \sigma_{i j} d \Omega-\int_{\Gamma_{t}} \delta u_{i} \bar{t}_{i} d \Gamma=0 & \forall \delta u_{i} \in \mathbb{H}_{0}^{1}(\Omega), \\
\int_{\Omega} \delta p\left(\varepsilon_{k k}+\frac{p}{\lambda}\right) d \Omega & =0 & \forall \delta p \in \mathbb{H}^{0}(\Omega) .
\end{array}
$$




\subsection{Discrete weak form}

In the standard displacement/pressure mixed formulation, the discretization procedure of the weak form yields a system of linear equations where both displacement and pressure parameter are part of the unknown vector. Our approach is distinct: starting from the weak form given in Eq. (10), we construct a displacement-based weak form such that the pressure approximation is obtained a posteriori from the displacement field. To this end, let us discretize the pressure parameter $p$ using linear finite element shape functions over a two-dimensional mesh of triangles:

$$
p^{h}(\mathbf{x})=\sum_{a=1}^{3} N_{a}(\mathbf{x}) p_{a}
$$

where $p_{a}$ are nodal pressures. ${ }^{2}$ The displacement is discretized using maximumentropy basis functions:

$$
\mathbf{u}^{h}(\mathbf{x})=\sum_{a=1}^{N} \phi_{a}(\mathbf{x}) \mathbf{u}_{a}
$$

which yields the following expression for the volumetric strain:

$$
\varepsilon_{k k}^{h}(\mathbf{x})=\left[\begin{array}{lll}
1 & 1 & 0
\end{array}\right] \sum_{a=1}^{N} \mathbf{B}_{a}(\mathbf{x}) \mathbf{u}_{a}=\mathbf{m}^{\mathrm{T}} \sum_{a=1}^{N} \mathbf{B}_{a}(\mathbf{x}) \mathbf{u}_{a},
$$

where $\mathbf{u}_{a}$ are nodal displacement coefficients and $\mathbf{B}_{a}(\mathbf{x})$ is the strain matrix for node $a$ :

$$
\mathbf{B}_{a}(\mathbf{x})=\left[\begin{array}{cc}
\phi_{a, x} & 0 \\
0 & \phi_{a, y} \\
\phi_{a, y} & \phi_{a, x}
\end{array}\right]
$$

\footnotetext{
${ }^{2}$ Although meshfree shape functions could potentially be used here, finite element shape functions are used for simplicity. Since no shape function derivatives of this field is required, poor tessellations or large distortions is not likely to drastically affect results.
} 
In order to ensure stability of the solution [1], the displacement approximation is enhanced with an extra displacement node in the interior of each triangle. This approach is similar to the use of nodal bubble shape functions in finite element methods [60], even though in the present case the max-ent basis function of the interior node does not necessarily vanish on the boundary of the triangle. See Ref. [61] for a related study on meshfree methods involving bubble functions. On substituting Eqs. (11) and (13) into Eq. (10), and relying on the arbitrariness of nodal pressure variations yields

$$
\sum_{b=1}^{N} \int_{\Omega} N_{a} \mathbf{m}^{\mathrm{T}} \mathbf{B}_{b} \mathbf{u}_{b} d \Omega+\frac{1}{\lambda} \sum_{b=1}^{3} \int_{\Omega} N_{a} N_{b} p_{b} d \Omega=0,
$$

and performing row-sum in the pressure term leads to

$$
\sum_{b=1}^{N}\left\{\int_{\Omega} N_{a} \mathbf{m}^{\mathrm{T}} \mathbf{B}_{b} d \Omega\right\} \mathbf{u}_{b}+\left\{\frac{1}{\lambda} \int_{\Omega} N_{a} d \Omega\right\} p_{a}=0 .
$$

From Eq. (16), we obtain the nodal pressure as

$$
p_{a}=-\lambda \sum_{b=1}^{N}\left\{\frac{\int_{\Omega} N_{a} \mathbf{m}^{\mathrm{T}} \mathbf{B}_{b} d \Omega}{\int_{\Omega} N_{a} d \Omega}\right\} \mathbf{u}_{b}
$$

which we refer to as volume-averaged nodal pressure. For the purpose of computation of integrals in Eq. (17), $\Omega$ is the union of all the elements attached to node $a$, i.e., $\Omega=\cup \Omega_{a}^{e}$.

Even though our approach shares common features with the method proposed by Krysl and Zhu [48], there exist notable differences. We use averages of strain matrices from the elements attached to a particular node to satisfy the near-incompressibility constraint in the weak form (10), whereas in Ref. [48] the averages are used to obtain a strain field that satisfies a 


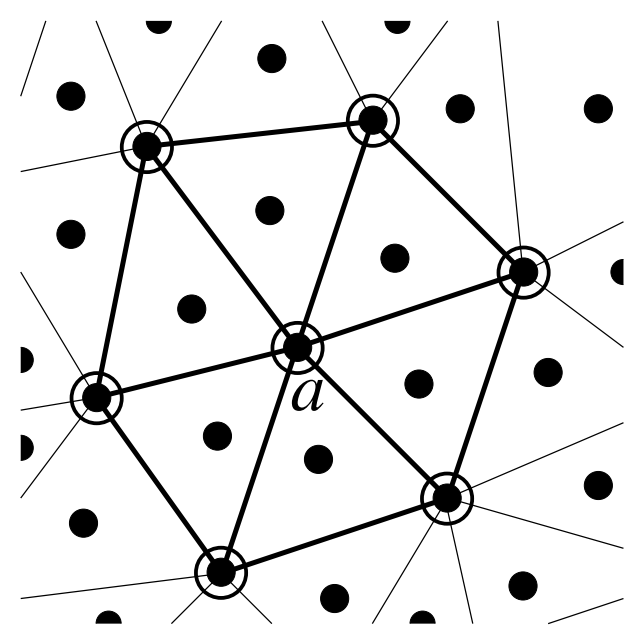

Fig. 2: Mesh to compute volume-averaged nodal pressure around a representative node $a$. Filled black circles represent displacement nodes and open circles are for pressure nodes.

kinematic constraint in a displacement-based weak form within a nodal integration scheme. A reference mesh for our proposed method is illustrated in Fig. 2.

Now, substituting Eqs. (11) and (12) into Eq. (7e), the following discretized material constitutive relation is obtained:

$$
\boldsymbol{\sigma}=-\mathbf{m} \sum_{a=1}^{3} N_{a} p_{a}+\overline{\mathbf{C}} \sum_{a=1}^{N} \mathbf{B}_{a} \mathbf{u}_{a}
$$

where

$$
\overline{\mathbf{C}}=\left[\begin{array}{ccc}
2 \mu & 0 & 0 \\
0 & 2 \mu & 0 \\
0 & 0 & \mu
\end{array}\right]
$$

and $p_{a}$ is given by Eq. (17). Finally, on substituting Eqs. (12) and (18a) into the weak form (10), and appealing to the arbitrariness of nodal variations, 
the following discrete system of equations is obtained:

$$
\mathrm{Kd}=\mathrm{f},
$$

where $\mathbf{d}$ is the vector of nodal coefficients and

$$
\begin{aligned}
\mathbf{K}_{a b} & =\int_{\Omega} \mathbf{B}_{a}^{\mathrm{T}} \overline{\mathbf{C}} \mathbf{B}_{b} d \Omega-\int_{\Omega} \mathbf{B}_{a}^{\mathrm{T}} \mathbf{m}\left\{\sum_{c=1}^{3} N_{c} \mathbf{Q}_{c b}\right\} d \Omega, \\
\mathbf{f}_{a} & =\int_{\Gamma_{t}} \phi_{a} \overline{\mathbf{t}} d \Gamma
\end{aligned}
$$

with

$$
\mathbf{Q}_{c b}=-\lambda\left\{\frac{\int_{\Omega} N_{c} \mathbf{m}^{\mathrm{T}} \mathbf{B}_{b} d \Omega}{\int_{\Omega} N_{c} d \Omega}\right\} .
$$

Note that owing to Eq. (17) in conjunction with Eq. (18), Eq. (19) depends only on the displacement field. The pressure field $p$ can be obtained a posteriori from the displacement field through Eq. (17).

\section{Modified Gauss integration}

As in finite element methods, numerical integration is used in meshfree methods to evaluate the weak form integrals that appear in Eq. (19). Typically, the support of meshfree basis functions is greater than the support of finite element basis functions, which lends flexibility to meshfree methods and often leads to improved accuracy. However, this has its consequences: with polynomial finite element basis functions whose support includes the union of triangles in a two-dimensional Delaunay tessellation, appropriate Gauss quadrature rules can be selected to ensure accurate and optimally convergent finite element solutions. In meshfree methods, these properties are lost, 
and hence use of standard Gauss quadrature to evaluate Eq. (19) leads to errors in numerical integration. To overcome this deficiency in existing meshfree methods, we devise a numerical integration scheme that alleviates the aforementioned errors.

\subsection{Modified strain}

We present a suitable modification to the standard Gauss quadrature to alleviate integration errors in meshfree methods and ensure patch test satisfaction to within machine precision. We propose the following modification to the weak form (10):

$$
\begin{aligned}
\int_{\Omega} \delta \hat{\varepsilon}_{i j} \sigma_{i j} d \Omega-\int_{\Gamma_{t}} \delta u_{i} \bar{t}_{i} d \Gamma=0 & \forall \delta u_{i} \in \mathbb{H}_{0}^{1}(\Omega) \\
\int_{\Omega} \delta p\left(\hat{\varepsilon}_{k k}+\frac{p}{\lambda}\right) d \Omega=0 & \forall \delta p \in \mathbb{H}^{0}(\Omega) .
\end{aligned}
$$

where $\hat{\varepsilon}$ bears resemblance to an assumed strain [49], which we refer in this paper as the modified strain. The modified strain $\hat{\varepsilon}$ introduces a correction in the evaluation of the stiffness matrix that alleviates integration errors in meshfree methods.

Let us consider the following modified strain in a certain background finite element cell:

$$
\hat{\varepsilon}=\varepsilon-\bar{\varepsilon}+\overline{\bar{\varepsilon}}
$$

where $\varepsilon$ is the standard small strain tensor, $\bar{\varepsilon}$ is the volume average strain tensor over the background cell, and $\overline{\bar{\varepsilon}}$ corresponds to $\bar{\varepsilon}$ written as a surface 
integral by means of Green's theorem. The corresponding equations are

$$
\begin{aligned}
\varepsilon & =\frac{1}{2}[\mathbf{u} \otimes \nabla+\nabla \otimes \mathbf{u}], \\
\bar{\varepsilon} & =\frac{1}{V^{e}} \int_{\Omega^{e}} \varepsilon d \Omega, \\
\overline{\bar{\varepsilon}} & =\frac{1}{V^{e}} \int_{\Gamma^{e}} \frac{1}{2}[\mathbf{u} \otimes \mathbf{n}+\mathbf{n} \otimes \mathbf{u}] d \Gamma .
\end{aligned}
$$

In the numerical examples that are presented in this paper, we refer to the integral in Eq. (22b) as the volume integral and the integral in Eq. (22c) as the surface integral. When linearly complete three-node or four-node finite elements are used, Eqs. (22b) and (22c) yield the same result with $\bar{\varepsilon}=\overline{\bar{\varepsilon}}$, and the small strain tensor is recovered. However, in the case of meshfree basis functions, this is in general not true. Indeed, if the support of the basis functions do not coincide with the background cell, Eqs. (22b) and (22c) yield different results. It is pertinent to mention here that the strain in the form of Eqs. (22b) and (22c) has been previously used in nodal integration schemes $[20,21,22]$, which in part has motivated the definition of the modified strain via Eq. (21).

On using $n$-point Gauss quadrature in the numerical integration of the weak form (20), the evaluation of $\hat{\varepsilon}$ will be required at each of these Gauss points $\mathbf{x}_{k}$, namely

$$
\hat{\varepsilon}\left(\mathbf{x}_{k}\right)=\varepsilon\left(\mathbf{x}_{k}\right)-\bar{\varepsilon}\left(\mathbf{x}_{k}\right)+\overline{\bar{\varepsilon}}\left(\mathbf{x}_{k}\right)
$$

Since $\bar{\varepsilon}$ and $\overline{\bar{\varepsilon}}$ are integral expressions over the background cell where numerical integration is carried out, it follows that for each $\mathbf{x}_{k}$ of the $n$-point evaluations, these integrals must also be computed using numerical integra- 
tion. ${ }^{3}$ In this approach, the integration order is preserved and the integration error is minimized when the same Gauss quadrature rule is used to integrate $\varepsilon$ as well as $\bar{\varepsilon}$. When the strain is a constant, which occurs in the patch test, machine precision accuracy is realized; see the proof in Appendix A.

\subsection{Three-node triangle}

Consider a three-node triangular background cell where the strain is computed as per Eq. (21). The approximation for the displacement field is: $\mathbf{u}^{h}(\mathbf{x})=\sum_{a=1}^{N} \phi_{a}(\mathbf{x}) \mathbf{u}_{a}$, and the discretized strains are:

$$
\begin{aligned}
& \varepsilon^{h}(\mathbf{x})=\sum_{a=1}^{N} \mathbf{B}_{a}(\mathbf{x}) \mathbf{u}_{a}, \\
& \overline{\boldsymbol{\varepsilon}}^{h}(\mathbf{x})=\frac{1}{A^{e}} \sum_{a=1}^{N}\left\{\sum_{p=1}^{n} \mathbf{B}_{a}\left(\mathbf{x}_{p}\right) A^{e} w_{p}\right\} \mathbf{u}_{a} \equiv \sum_{a=1}^{N} \overline{\mathbf{B}}_{a} \mathbf{u}_{a}, \\
& \overline{\bar{\varepsilon}}^{h}(\mathbf{x})=\sum_{a=1}^{N}\left\{\frac{1}{A^{e}} \sum_{L=1}^{3}\left[\sum_{r=1}^{m} \overline{\overline{\mathbf{N}}}_{a}\left(\xi_{r}\right)\left|J\left(\xi_{r}\right)\right| w_{r}\right]\right\} \mathbf{u}_{a} \equiv \sum_{a=1}^{N} \overline{\overline{\mathbf{B}}}_{a} \mathbf{u}_{a},
\end{aligned}
$$

and in two dimensions

$$
\mathbf{B}_{a}(\mathbf{x})=\left[\begin{array}{cc}
\phi_{a, x} & 0 \\
0 & \phi_{a, y} \\
\phi_{a, y} & \phi_{a, x}
\end{array}\right], \quad \overline{\mathbf{N}}_{a}=\left[\begin{array}{cc}
\phi_{a} n_{x} & 0 \\
0 & \phi_{a} n_{y} \\
\phi_{a} n_{y} & \phi_{a} n_{x}
\end{array}\right] .
$$

In Eq. (24d), $n_{x}$ and $n_{y}$ are the $x$ and $y$-component of the unit outward normal to the cell's edge, respectively. Furthermore, $n$-point and $m$-point Gauss quadrature rules have been used for numerical integration of the volume integral and surface integral, respectively; and $A^{e}$ is the element area

\footnotetext{
${ }^{3}$ Note that $\bar{\varepsilon}$ and $\overline{\bar{\varepsilon}}$ are constants within each background cell, and hence are precomputed.
} 
(uniform thickness is assumed). Discretized strains are then used to write the discretized modified strain tensor as follows:

$$
\hat{\varepsilon}^{h}(\mathbf{x})=\sum_{a=1}^{N} \hat{\mathbf{B}}_{a}(\mathbf{x}) \mathbf{u}_{a}
$$

with

$$
\hat{\mathbf{B}}_{a}(\mathbf{x})=\mathbf{B}_{a}(\mathbf{x})-\mathbf{G}_{a}
$$

and

$$
\mathbf{G}_{a}=\sum_{p=1}^{n} \mathbf{B}_{a}\left(\mathbf{x}_{p}\right) w_{p}-\frac{1}{A^{e}} \sum_{L=1}^{3}\left\{\sum_{r=1}^{m} \overline{\overline{\mathbf{N}}}_{a}\left(\xi_{r}\right)\left|J\left(\xi_{r}\right)\right| w_{r}\right\} .
$$

4.3. Numerical integration of the stiffness matrix and the external force vector

In Eq. (19), matrix $\mathbf{K}_{a b}$ now appears corrected in terms of the modified strain matrix $\hat{\mathbf{B}}_{a}$, and is numerically integrated using $n$-point Gauss quadrature rule. Recall that the same Gauss quadrature rule is used in Eq. (22b). In particular, for a three-node triangular background cell, the numerical integration of the stiffness matrix disregarding the pressure part is computed as follows:

$$
\mathbf{K}_{a b}=\sum_{k=1}^{n} \hat{\mathbf{B}}_{a}^{\mathrm{T}}\left(\mathbf{x}_{k}\right) \overline{\mathbf{C}} \hat{\mathbf{B}}_{b}\left(\mathbf{x}_{k}\right) A^{e} t w_{k}
$$

where $A^{e}$ is the area of the three-node triangle and $t$ its thickness. In Eq. (26), indices $a$ and $b$ range over the nodes covered by the intersection of the support of the basis functions contained in $\hat{\mathbf{B}}_{a}$ and $\hat{\mathbf{B}}_{b}$. Numerical integration of the external force vector is done as usual with an $n$-point Gauss quadrature rule. 


\subsection{On the selection of the appropriate Gauss quadrature rule}

The weak form integrals appearing in Eq. (20) need to be computed with sufficient accuracy to preclude underintegration or a rank-deficient stiffness matrix. Due to the interior displacement node that is added inside the triangle for stability, at least three Gauss points are needed to compute the volume integrals, which is confirmed by the numerical experiments presented in Section 5. Using a 1-point quadrature rule will lead to a rank-deficient stiffness matrix. On the other hand, the computation of the surface integral is not a significant issue since it does not involve basis function derivatives; we use a 2-point quadrature rule on each edge. The above mentioned quadrature rules suffice to pass the patch test to machine precision and to ensure optimal rates of convergence in the energy norm for the proposed meshfree method.

\section{Numerical examples}

We study the accuracy and performance of the maximum-entropy meshfree (MEM) method by means of four two-dimensional benchmark problems: displacement patch test, cantilever beam subjected to a parabolic end load, Cook's membrane problem, and a rigid flat punch under frictionless indentation. In all the problems other than the patch test we compare the maximum-entropy solution to a finite element solution (MINI element). In the numerical examples, we use the acronyms STD to refer to standard Gaussian integration and MOD for modified Gaussian integration (see Section 4). Unless stated otherwise, we use MOD with 3-point Gauss quadrature for the volume integral and 2-point Gauss quadrature for the surface integral in the 
MEM computations. It is reminded that the implementation uses only three stress points per triangle; the surface Gauss points only sample displacement and are used to modify the strain at these stress points.

\subsection{Displacement patch test}

Consider the boundary-value problem for a two-dimensional elastic plate under essential boundary conditions:

$$
\begin{gathered}
\boldsymbol{\nabla} \cdot \boldsymbol{\sigma}=\mathbf{0} \quad \text { in } \Omega=(0,1)^{2}, \\
u_{x}(\mathbf{x})=x \quad \text { on } \Gamma, \quad u_{y}(\mathbf{x})=x+y \quad \text { on } \Gamma .
\end{gathered}
$$

Plane strain conditions are assumed with the following material parameters: $E=3 \times 10^{7}$ and $\nu=\{0.3 ; 0.499\}$. The meshes used in the study are shown in Fig. 3: a uniform mesh of four-node quadrilateral elements (Q4) for $\nu=0.3$, and a non-uniform mesh of three-node triangular elements (T3) for $\nu=\{0.3 ; 0.499\}$. Both meshes are tested using STD and MOD schemes. Maximum-entropy basis functions are used with a support-width parameter $\gamma=2.0$ for the Gaussian prior, and $\gamma=1.5$ for the quartic prior. Numerical results for the relative error in the $L^{2}$-norm are shown in Tables 1 and 2 . Different Gauss quadrature rules for the volume integrals are tested (quadrature rule for quadrilateral elements is indicated within braces). Numerical results confirm that patch test satisfaction is met to within machine precision for both compressible and near-incompressible material behavior only when MOD is employed. In this study, max-ent approximants are used, but the generality of the integration approach renders it applicable to other meshfree approximants as well as polygonal finite element interpolants [62]. 


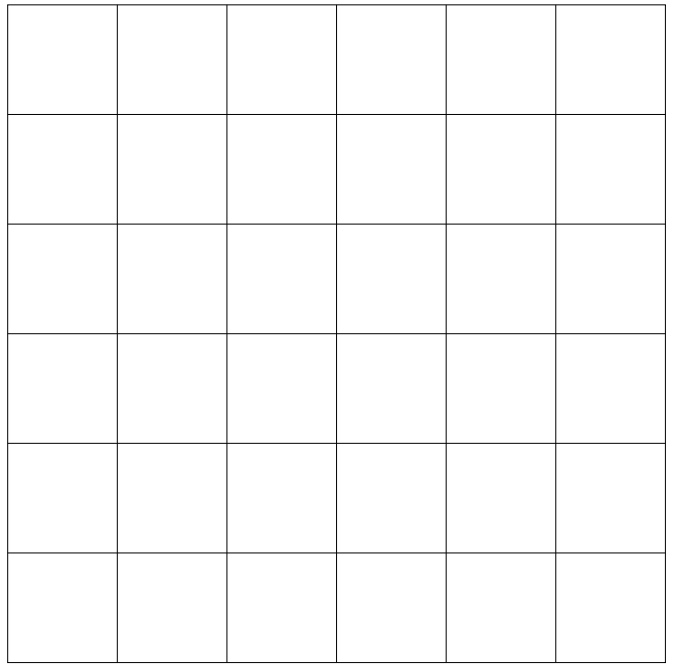

(a)

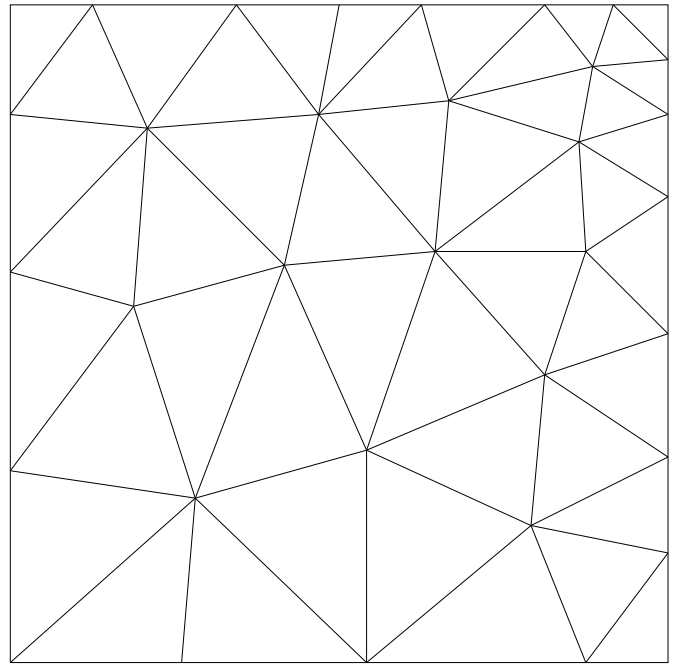

(b)

Fig. 3: Meshes used for the displacement patch test. (a) Uniform mesh of four-node quadrilaterals (Q4); and (b) Non-uniform mesh of three-node triangles (T3). For the near-incompressible case, nodal degrees of freedom are as shown in Fig. 2.

Table 1: Relative error in the $L^{2}$-norm for the patch test $(\nu=0.3)$

\begin{tabular}{|c|c|c|c|c|c|}
\hline Prior & Quadrature & T3 $(\mathrm{STD})$ & T3 $(\mathrm{MOD})$ & Q4 $(\mathrm{STD})$ & Q4 (MOD) \\
\hline \multirow{4}{*}{ Gaussian } & $3(3 \times 3)$ & $1.7 \times 10^{-3}$ & $3.2 \times 10^{-16}$ & $6.4 \times 10^{-6}$ & $1.2 \times 10^{-15}$ \\
& $6(6 \times 6)$ & $5.6 \times 10^{-4}$ & $3.1 \times 10^{-16}$ & $1.9 \times 10^{-8}$ & $2.8 \times 10^{-15}$ \\
& $12(12 \times 12)$ & $2.9 \times 10^{-4}$ & $3.4 \times 10^{-16}$ & $6.6 \times 10^{-12}$ & $2.5 \times 10^{-15}$ \\
\hline \multirow{3}{*}{ Quartic } & $3(3 \times 3)$ & $2.6 \times 10^{-3}$ & $2.8 \times 10^{-16}$ & $1.3 \times 10^{-4}$ & $3.2 \times 10^{-16}$ \\
& $6(6 \times 6)$ & $3.0 \times 10^{-3}$ & $4.4 \times 10^{-16}$ & $5.6 \times 10^{-7}$ & $9.3 \times 10^{-16}$ \\
& $12(12 \times 12)$ & $7.8 \times 10^{-4}$ & $3.6 \times 10^{-16}$ & $1.3 \times 10^{-8}$ & $7.9 \times 10^{-16}$ \\
\hline
\end{tabular}


Table 2: Relative error in the $L^{2}$-norm for the patch test $(\nu=0.499)$

\begin{tabular}{|c|c|c|c|}
\hline Prior & Quadrature & T3 (STD) & T3 (MOD) \\
\hline \multirow{3}{*}{ Gaussian } & 3 & $5.4 \times 10^{-1}$ & $8.2 \times 10^{-14}$ \\
& 6 & $4.8 \times 10^{-1}$ & $8.8 \times 10^{-14}$ \\
& 12 & $4.5 \times 10^{-1}$ & $8.6 \times 10^{-14}$ \\
\hline \multirow{2}{*}{ Quartic } & 3 & $5.2 \times 10^{-1}$ & $2.6 \times 10^{-13}$ \\
& 6 & $3.9 \times 10^{-1}$ & $2.6 \times 10^{-13}$ \\
& 12 & $5.1 \times 10^{-1}$ & $6.2 \times 10^{-13}$ \\
\hline
\end{tabular}

\subsection{Cantilever beam}

We consider the cantilever beam of thickness $t$ with a a parabolic end load $P$ (Fig. 4(a)). The displacement solution for compressible $(\nu=0.3)$ and near-incompressible $(\nu=0.499999)$ material behavior with $E=10^{7}$ in plane strain condition is sought. Essential boundary conditions on the clamped edge are applied according to the analytical solution given by Timoshenko and Goodier [63]:

$$
\begin{aligned}
& u_{x}=\frac{P y}{6 \bar{E} I}\left(3 x^{2}-6 L x+\bar{\nu} y^{2}\right)-\frac{P y}{6 I \mu}\left(y^{2}-\frac{3}{4} D^{2}\right), \\
& u_{y}=\frac{P}{6 \bar{E} I}\left(3 \bar{\nu}(L-x) y^{2}+3 L x^{2}-x^{3}\right)
\end{aligned}
$$

where $\mu$ is the material shear modulus (Lamé parameter) and

$$
\begin{aligned}
& \bar{E}=\left\{\begin{array}{ll}
E & \text { for plane stress } \\
E /\left(1-\nu^{2}\right) & \text { for plane strain }
\end{array},\right. \\
& \bar{\nu}= \begin{cases}\nu & \text { for plane stress } \\
\nu /(1-\nu) & \text { for plane strain }\end{cases}
\end{aligned}
$$


In the numerical computations the following parameters are used: $L=16$, $D=4, t=1$ and $P=-1$. Two background meshes for the upper half of the beam are studied: a regular mesh of three-node triangles (Fig. 4(b)) and an irregular mesh of three-node triangles (Fig. 4(c)). Maximum-entropy basis functions are used with a support-width parameter $\gamma=2.0$ for the Gaussian prior. The numerical solution of the maximum-entropy meshfree method with MOD is compared to the finite element solution (MINI element [64]) for the standard displacement/pressure mixed formulation. Results for the normalized tip deflection are shown in Table 3 for both meshes. The numerical and exact solution for the nodal hydrostatic pressure along the fibers of the beam (regular mesh only) are depicted in Fig. 5(a) for the MINI element and in Fig. 5(b) for the maximum-entropy meshfree method. For the pressure field, the MINI element has some oscillations about the analytical solution, whereas the maximum-entropy solution is devoid of oscillations and is in very good agreement with the analytical solution. The convergence study of the normalized tip deflection for the regular mesh is shown in Fig. 6 for the MINI element and the MEM method. The numerical results indicate that compared to the finite element solution, the MEM solution has better accuracy and converges faster towards the exact tip-deflection.

To assess the influence of numerical integration, a study of the MEM method with STD and MOD schemes is conducted. The numerical results for $\nu=0.3$ and $\nu=0.499999$ are presented in Fig. 7. For $\nu=0.3$, the standard displacement-based max-ent formulation is used with nodes located only at the vertices of the triangles. From the convergence curves in Fig. 7(a), we observe that the rate of convergence of STD (with 3-, 6-, and 12-point 


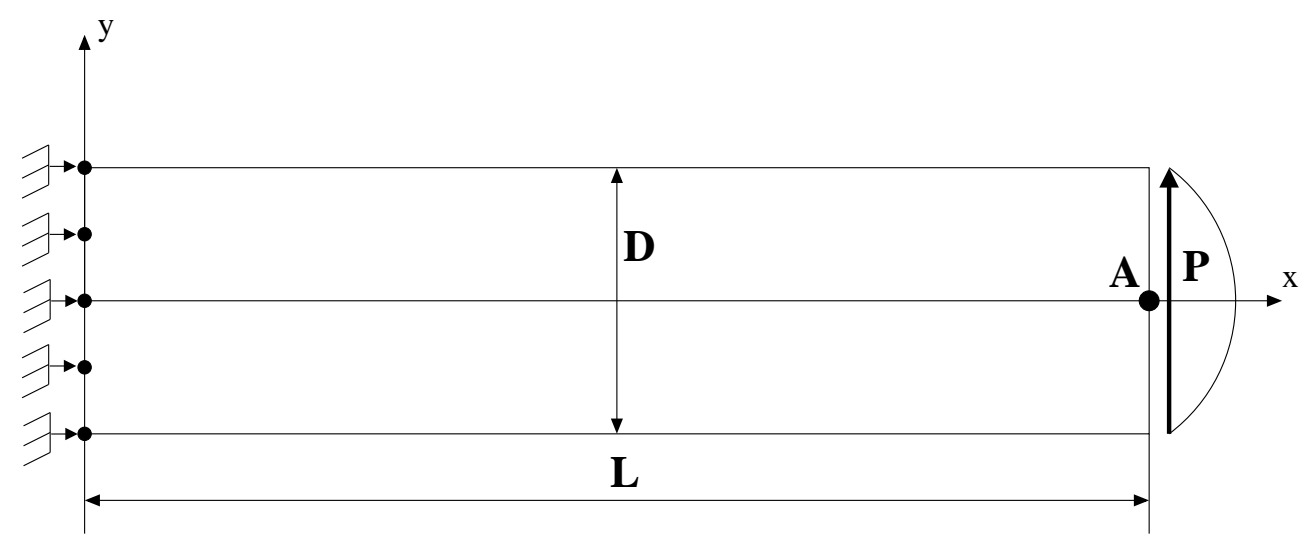

(a)

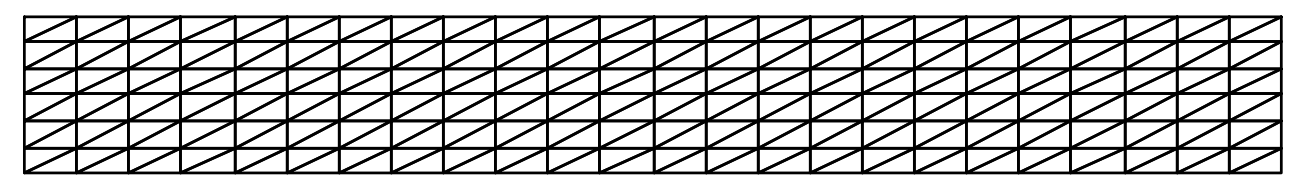

(b)

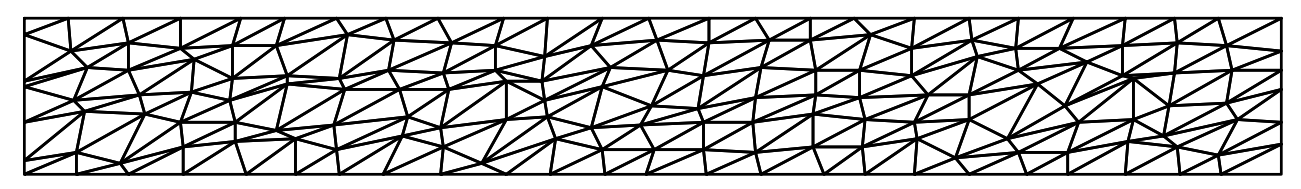

(c)

Fig. 4: Cantilever beam problem. (a) Model geometry and boundary conditions; (b) Regular mesh of three-node triangles; and (c) Irregular mesh of three-node triangles. Unless otherwise stated in the text, nodal degrees of freedom are as shown in Fig. 2 for both compressible and near-incompressible elasticity.

quadrature) and MOD techniques are in agreement with theory - the energy norm of the error is of $O(h)$. For $\nu=0.499999$, the nodal-averaged pressure formulation is adopted, and an additional displacement-node is inserted in 
Table 3: Normalized tip deflection for the cantilever beam (plane strain).

\begin{tabular}{|c|c|c|c|c|}
\hline \multirow{2}{*}{} & \multicolumn{3}{|c|}{$u_{2}^{\mathrm{NUM}} / u_{2}^{\mathrm{EXACT}}$ at point A } \\
\hline Method & $\nu=0.3$ & $\nu=0.499999$ & $\nu=0.3$ & $\nu=0.499999$ \\
\hline MINI & 0.963 & 0.969 & 0.955 & 0.962 \\
MEM & 1.001 & 0.999 & 1.001 & 1.000 \\
\hline
\end{tabular}

the middle of every triangle. It is evident from the curves in Fig. 7(b) that a 3-point Gauss quadrature is insufficient (under-integration leads to lack of convergence), and only with higher-order Gauss quadrature is the convergence rate closer to optimal. This is not surprising, since 3-point and 6-point quadrature rules in a triangle are exact for second-order and fourth-order bivariate polynomials, respectively, but the max-ent basis function for the interior node bears similarity to a cubic bubble function, which renders the integrand of the stiffness matrix to be like a fourth-order bivariate polynomial. Hence, the improved accuracy with 6-point quadrature is realized, with 12-point quadrature being able to deliver about the same accuracy as the modified integration scheme. The numerical results demonstrate the performance of STD and MOD schemes, and establishes that the MOD technique can deliver accurate and optimal convergence in MEM computations.

Lastly, the accuracy and rate of convergence of the MINI element and the maximum-entropy meshfree method for two support-width parameters $\gamma$ are compared in Fig. 8. For the Gaussian prior, $\gamma=2.0$ represents a larger support and $\gamma=4.0$ a smaller support. From Fig. 8, we observe that the max-ent and finite element solutions are accurate and have the optimal rate 


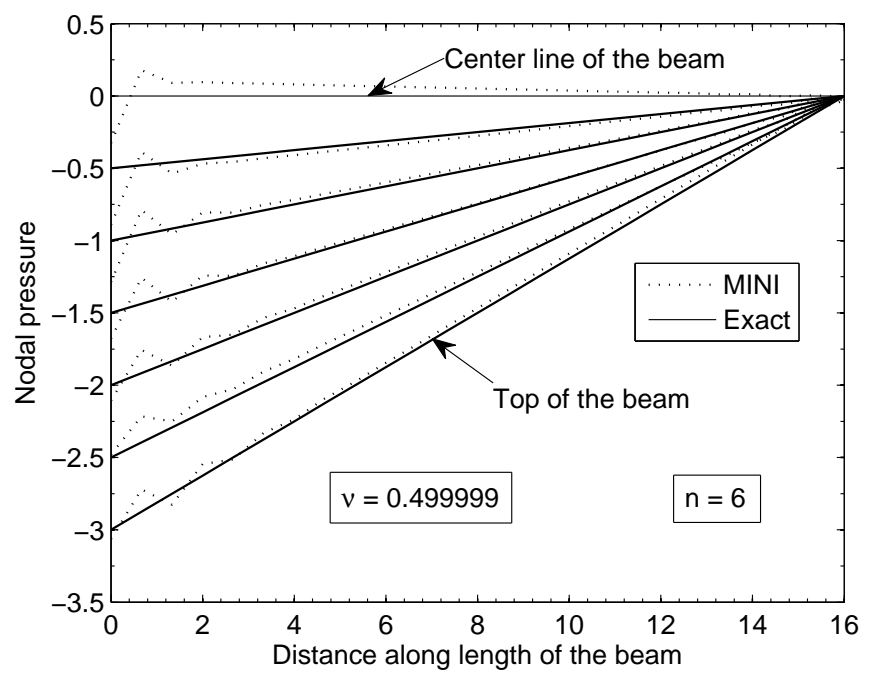

(a)

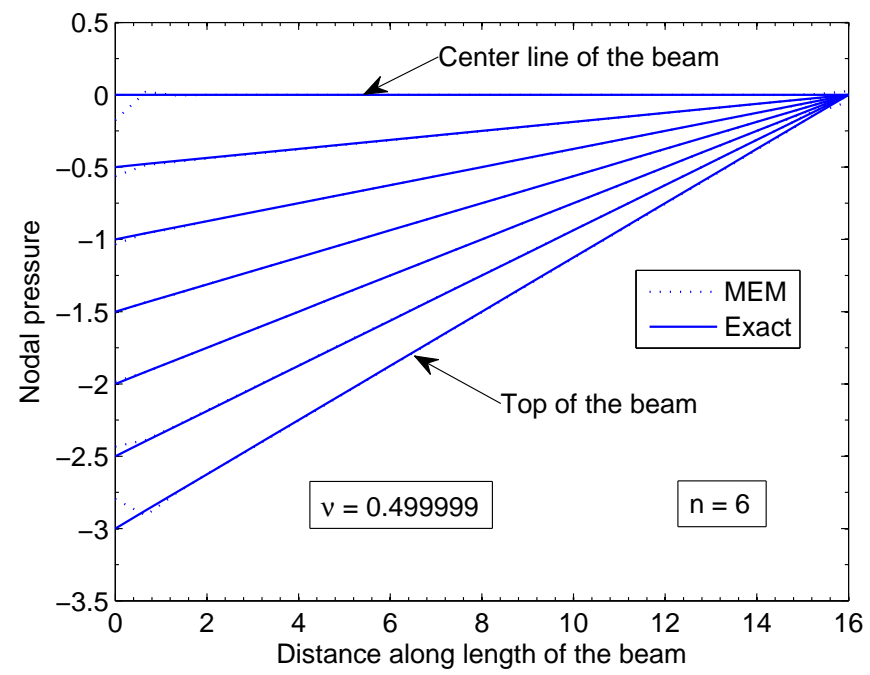

(b)

Fig. 5: Cantilever beam problem. (a) Nodal pressure for the MINI element; and (b) Nodal pressure for the maximum-entropy meshfree method. 


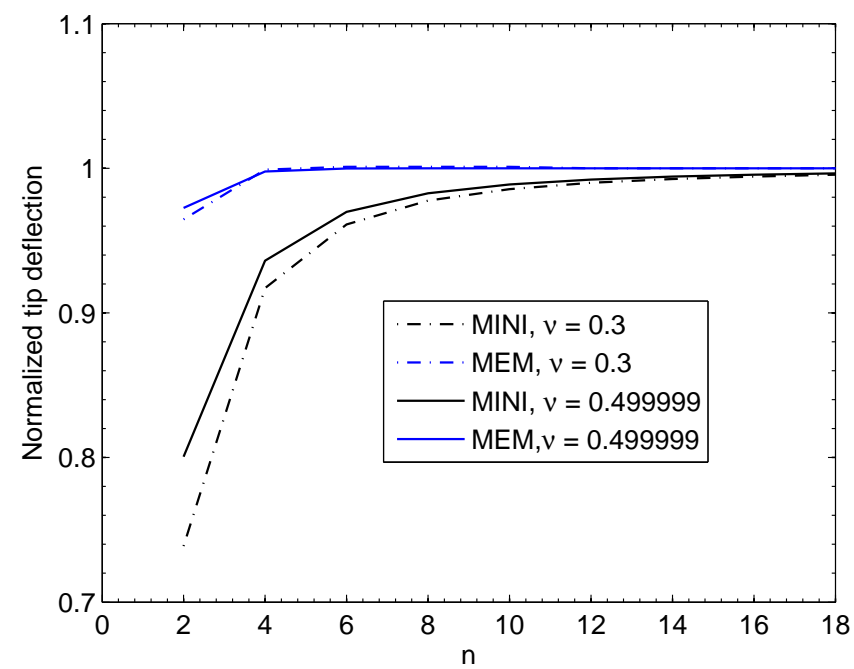

Fig. 6: Convergence of the normalized tip deflection for the cantilever beam problem. $4 n \times n$ ( $n$ is the number of divisions along the $y$-direction) mesh pattern is used on the upper half of the beam.

of convergence in the energy norm for both support sizes.

\subsection{Cook's membrane}

The model geometry and boundary conditions for the Cook's membrane problem is shown in Fig. 9(a). This standard benchmark problem is suitable to test the behavior of the near-incompressible formulation under combined bending and shear (see for instance, Refs. [4, 65, 66]). The left edge is clamped and the right end is subjected to a shear load $F=6.25$ per unit length (total shear load of 100). The following material parameters are considered: $E=250$ and $\nu=0.4999$. A regular mesh of three-node triangles is used with a mesh pattern of $n \times n$ divisions per side. A reference mesh for $n=6$ is shown in Fig. 9(b). Maximum-entropy basis functions are used with 


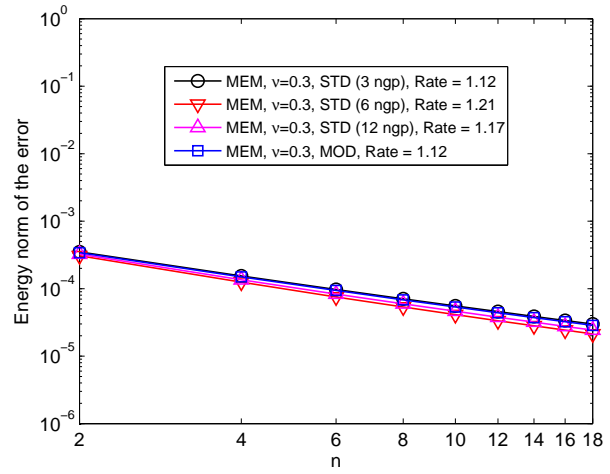

(a)

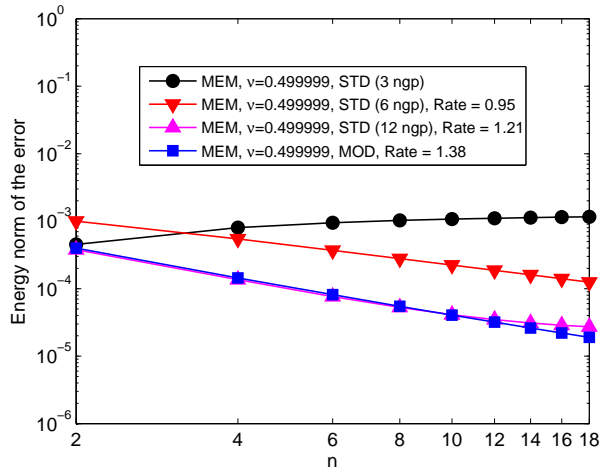

(b)

Fig. 7: Rate of convergence in energy norm for the cantilever beam problem using standard and modified Gauss integration techniques. (a) $\nu=0.3$; and (b) $\nu=0.499999 .4 n \times n$ ( $n$ is the number of divisions along the $y$-direction) mesh pattern is used on the upper half of the beam.

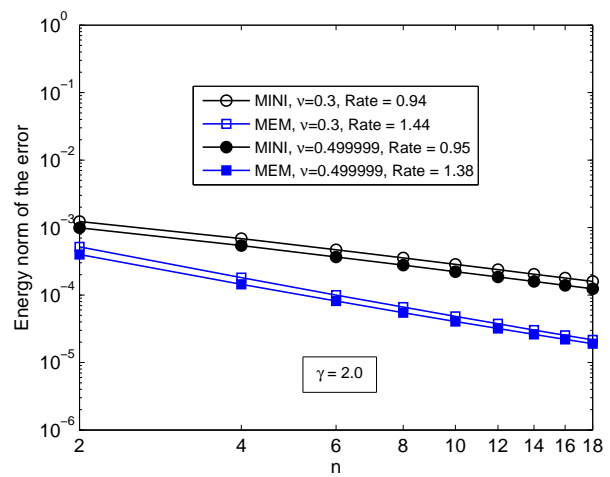

(a)

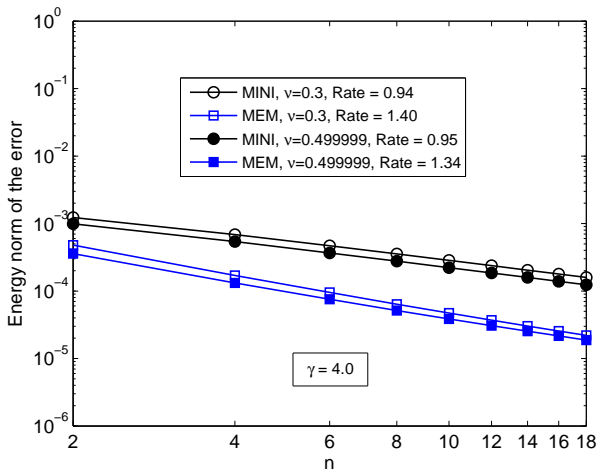

(b)

Fig. 8: Rate of convergence in energy norm for the cantilever beam problem. (a) $\gamma=2.0$; and (b) $\gamma=4.0 .4 n \times n$ ( $n$ is the number of divisions along the $y$-direction) mesh pattern is used on the upper half of the beam. 
a support-width parameter $\gamma=2.0$ for the Gaussian prior. The numerical solution of the maximum-entropy meshfree method with MOD is compared to the finite element solution (MINI element [64]) for the standard displacement/pressure mixed formulation. The convergence study of the vertical tip displacement at point A upon mesh refinement is shown in Fig. 9(c) for both the MINI element and the maximum-entropy meshfree method. Numerical results indicate that the max-ent solution has a faster convergence in the vertical tip displacement vis-à-vis the MINI element solution. We also observe that the MINI element produces oscillations in the hydrostatic pressure field, whereas the maximum-entropy pressure field is smooth.

\subsection{Rigid flat punch}

As the last numerical example, we study the deformation of a square block of dimensions $1 \times 1$ with unit thickness under plane strain state in response to a frictionless indentation. A similar benchmark problem has been considered in Refs. [65, 66, 67]. The bottom, left, and right edges are

fully clamped, which imposes a severe constraint on allowable deformation states when $\nu \rightarrow 0.5$. A downward displacement of 0.03 is applied over the center portion of the top edge covering $1 / 3$ of the edge's length (see Fig. 10). In the numerical computations the following parameters are specified: $E=10^{7}, \nu=0.499999$. Maximum-entropy basis functions are used with a support-width parameter $\gamma=1.5$ for the quartic prior. In Figs. 11(a) and 11(b), the numerical hydrostatic pressure field parameter is shown for the maximum-entropy meshfree method within the standard displacementbased formulation (no interior displacement node in the triangle) and for two different Gauss quadratures. As expected, severe volumetric locking occurs. 


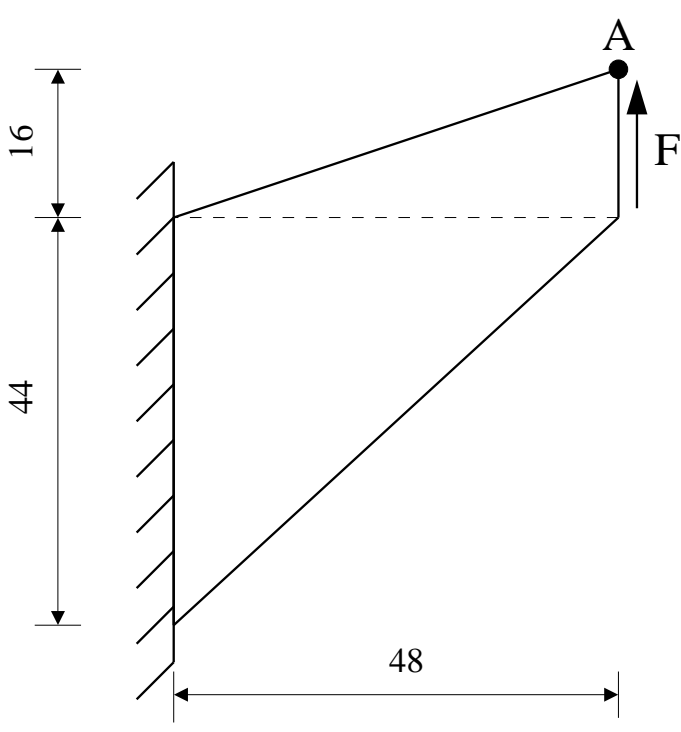

(a)

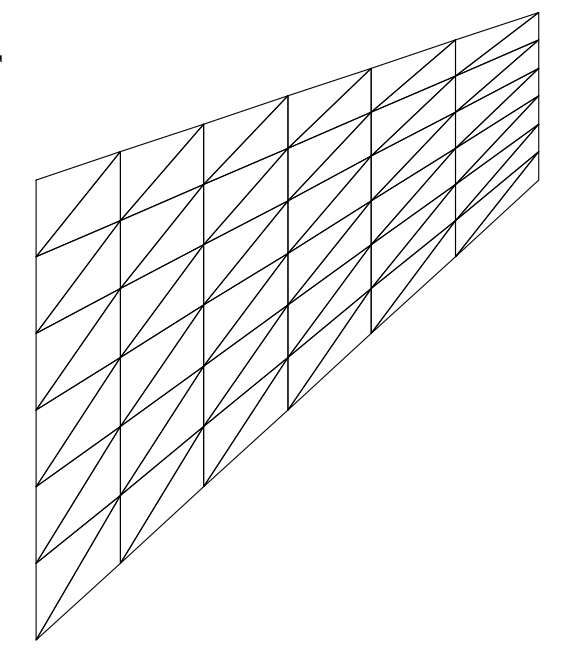

(b)

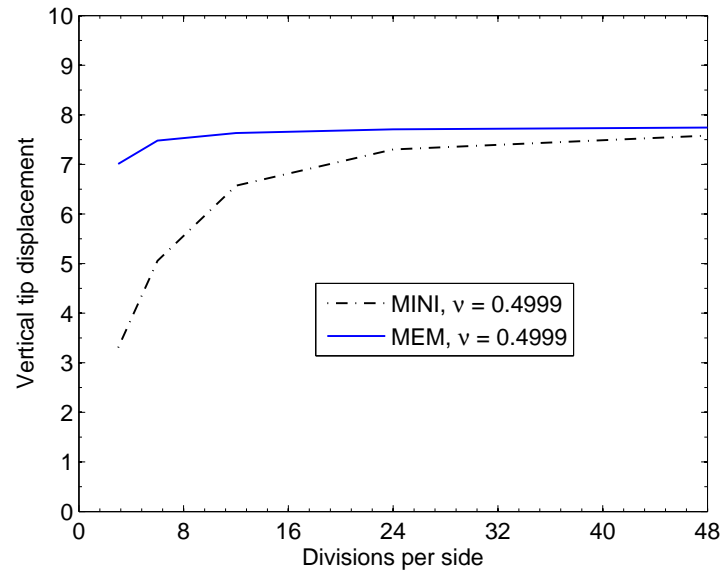

(c)

Fig. 9: Cook's membrane problem. (a) Model geometry and boundary conditions; (b) Sample mesh; and (c) Vertical tip displacement. Nodal degrees of freedom are as shown in Fig. 2. 


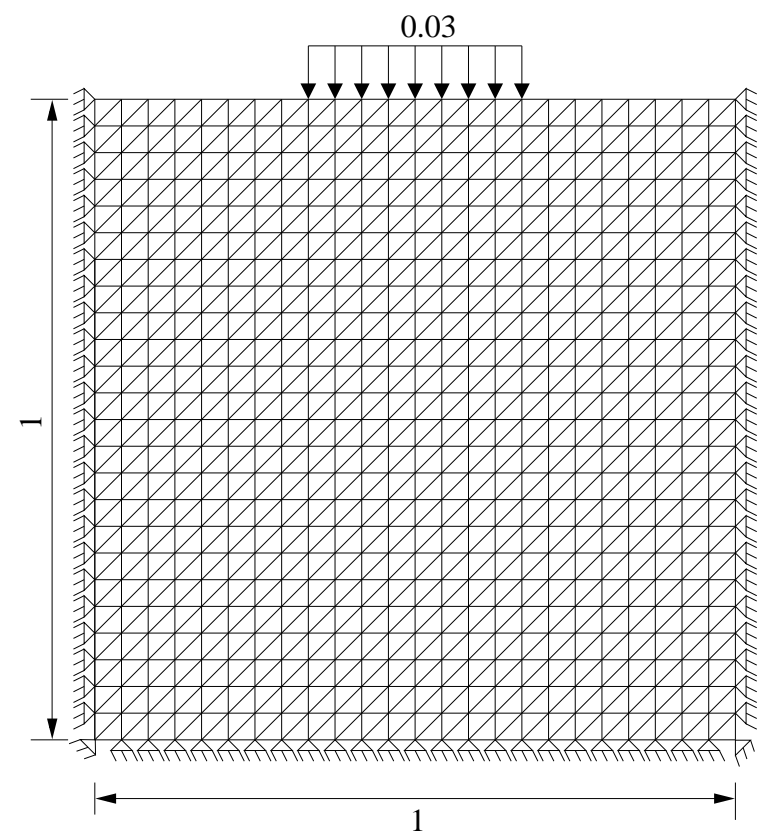

Fig. 10: Geometry and boundary conditions for the rigid flat punch problem. Unless otherwise stated in the text, nodal degrees of freedom are as shown in Fig. 2.

The corresponding maximum-entropy solution for the volume-averaged nodal pressure formulation is presented in Fig. 11(c), and we observe that the solution is free of volumetric locking and spurious checkerboarding modes. For comparison, the non-locking MINI element solution is presented in Fig. 11(d), which behaves less smooth than MEM solution.

In order to investigate the performance of the MEM solution on unstructured meshes, a test is conducted for the MEM method and the MINI element on the mesh depicted in Fig. 12a. Results for the pressure field solution are illustrated in Fig. 12b for the MEM method and in Fig. 12c for the MINI element solution. We observe that the MEM method provides a smooth 


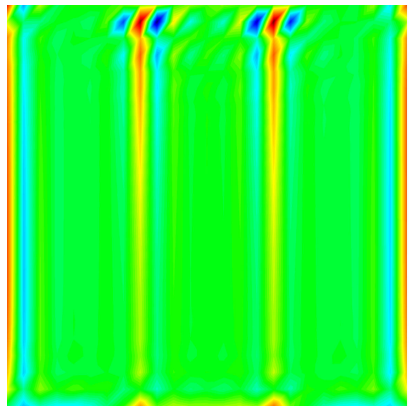

(a)

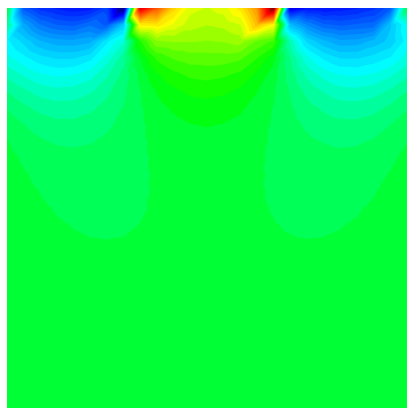

(c)

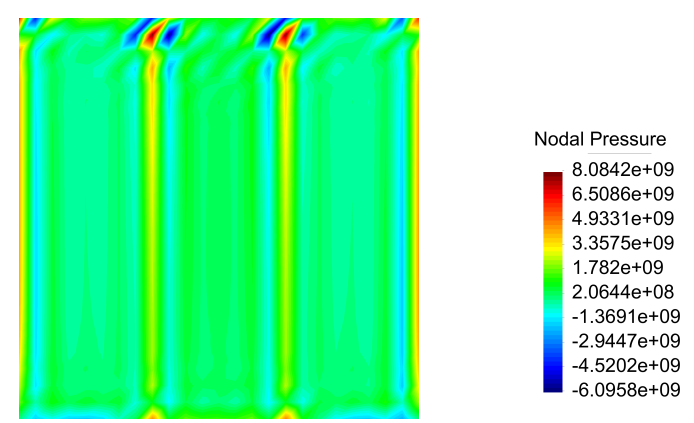

(b)

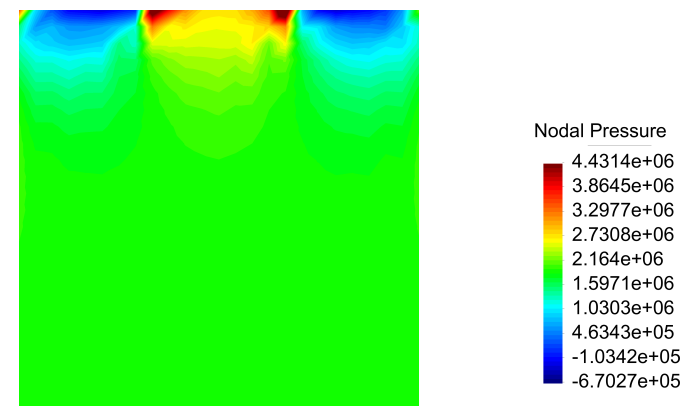

Fig. 11: Rigid flat punch problem. (a), (b) MEM method with displacementbased linear elasticity and standard 3-point and 12-point Gauss quadrature rules, respectively; and (c) MEM method with volume-averaged nodal pressure approach and modified integration technique; and (d) MINI element solution.

pressure field, whereas the MINI element solution is non-converged.

\section{Concluding remarks}

In this paper, a meshfree method for compressible and near-incompressible elasticity based on maximum-entropy approximants was presented. The adoption of maximum-entropy basis functions provides flexibility and eases 


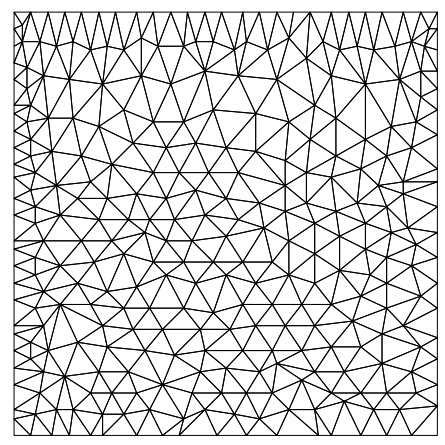

(a)

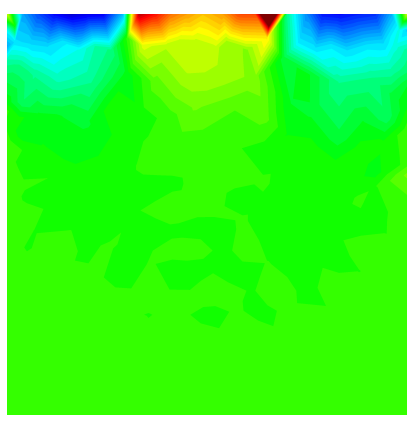

(b)

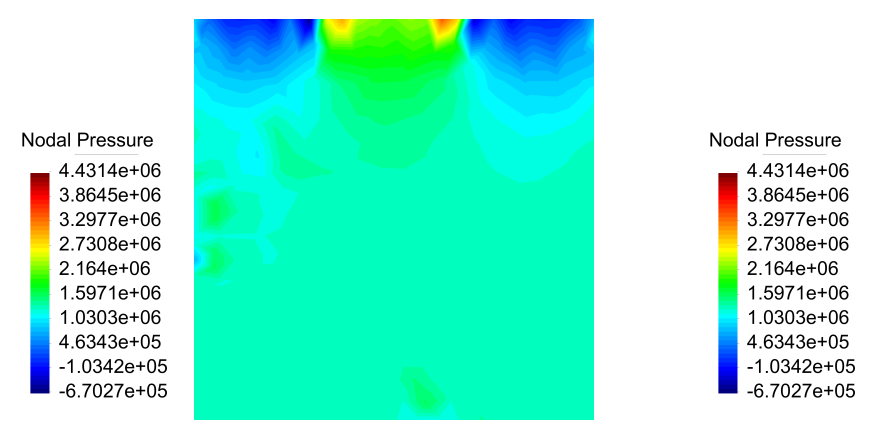

(c)

Fig. 12: Rigid flat punch problem. (a) Unstructured background mesh, (b) and (c) pressure field solution for the MEM method and MINI element, respectively.

the implementation since it permits the direct imposition of essential boundary conditions.

A modified Gaussian integration scheme was devised, which alleviated numerical integration errors in meshfree methods and satisfied the patch test to within machine precision. The procedure only required three stress points per triangle and was simple and robust. The new numerical integration scheme was employed in a meshfree formulation for near-incompressible material 
behavior. A standard displacement/pressure mixed formulation was used to compute volume-averaged nodal pressures a posteriori from the displacement field of surrounding nodes. Various benchmark problems in two dimensions, which included displacement patch test, near-incompressible analysis of a cantilever beam, Cook's membrane (combined bending and shear) and rigid flat punch under frictionless indentation were presented to demonstrate the performance of the maximum-entropy meshfree method for compressible and near-incompressible elasticity. The numerical results revealed that the maximum-entropy solution was devoid of volumetric locking and converged optimally in the energy norm. The accurate numerical results in two dimensional linear elasticity point to the potential of the maximum-entropy meshfree formulation in nonlinear and three-dimensional computations.

\section{Appendix A}

We prove that the modified Gauss quadrature scheme presented in Section 4 exactly satisfies the patch test on a background mesh of three-node triangles. To wit, it suffices to show that the nodal forces at all interior nodes (whose basis function support vanish on the boundary) are identically equal to zero for a uniform stress field $\boldsymbol{\sigma}=\boldsymbol{\sigma}^{c}$, i.e.,

$$
\mathbf{f}_{a}=\sum_{e} \int_{\Omega^{e}} \hat{\mathbf{B}}_{a}^{\mathrm{T}} \boldsymbol{\sigma}^{c} d \Omega=\mathbf{0}
$$

is to be established, where the assembly is over all elements $e$ that have a non-zero intersection with the support of $\phi_{a}$.

Proof. From Eqs. (24) and (25), we can write the matrix $\hat{\mathbf{B}}_{a}$ as

$$
\hat{\mathbf{B}}_{a}(\mathbf{x})=\mathbf{B}_{a}(\mathbf{x})-\overline{\mathbf{B}}_{a}+\overline{\overline{\mathbf{B}}}_{a}=\mathbf{B}_{a}(\mathbf{x})-\sum_{p=1}^{n} \mathbf{B}_{a}\left(\mathbf{x}_{p}\right) w_{p}+\overline{\overline{\mathbf{B}}}_{a}
$$


and hence $\mathbf{f}_{a}$ can be expressed as

$$
\mathbf{f}_{a}=\sum_{e} \int_{\Omega^{e}}\left[\mathbf{B}_{a}^{T}(\mathbf{x})-\sum_{p=1}^{n} \mathbf{B}_{a}^{T}\left(\mathbf{x}_{p}\right) w_{p}+\overline{\overline{\mathbf{B}}}_{a}^{T}\right] \boldsymbol{\sigma}^{c} d \Omega .
$$

On performing numerical integration using $n$-point Gauss quadrature within the element, we obtain

$$
\mathbf{f}_{a}=\sum_{e} \sum_{q=1}^{n}\left[\mathbf{B}_{a}^{T}\left(\mathbf{x}_{q}\right) w_{q}-\left(\sum_{p=1}^{n} \mathbf{B}_{a}^{T}\left(\mathbf{x}_{p}\right) w_{p}\right) w_{q}+\overline{\bar{B}}_{a}^{T} w_{q}\right] A^{e} \boldsymbol{\sigma}^{c},
$$

which simplifies to

$$
\mathbf{f}_{a}=\sum_{e} \sum_{q=1}^{n} \overline{\overline{\mathbf{B}}}_{a}^{T} A^{e} w_{q} \boldsymbol{\sigma}^{c}
$$

since the first two terms cancel because $\sum_{q=1}^{n} w_{q}=1$ (Gauss weights sum to unity). Recalling the expression for $\overline{\overline{\mathbf{B}}}_{a}$ from Eq. (25), we have

$$
\mathbf{f}_{a}=\sum_{e} \sum_{q=1}^{n} \sum_{L=1}^{3}\left\{\sum_{r=1}^{m} \overline{\overline{\mathbf{N}}}_{a}^{T}\left(\xi_{r}\right)\left|J\left(\xi_{r}\right)\right| w_{r}\right\} w_{q} \boldsymbol{\sigma}^{c}
$$

Now, closer inspection of the above equation and the expression for $\overline{\bar{N}}_{a}$ given in Eq. (24d) reveals that contribution along an interior edge $L$ will arise from two adjacent triangles with common edge $L$. However, since the normal vector on the edge will assume equal magnitude but opposite signs for the two cases, the two contributions cancel each other. Proceeding likewise, the net contribution to $\mathbf{f}_{a}$ from all interior edges vanishes, and hence Eq. (A.1) is satisfied.

\section{Acknowledgement}

The authors (A. Ortiz and N. Sukumar) gratefully acknowledge the research support of the National Science Foundation through contract grants CMMI-0626481 and CMMI-0826513 to the University of California at Davis. 
The work of M. A. Puso was performed under the auspices of the U.S. Department of Energy by Lawrence Livermore National Laboratory under Contract DE-AC52-07NA27344.

\section{References}

[1] T. J. R. Hughes, The Finite Element Method: Linear Static and Dynamic Finite Element Analysis, Dover Publications, Inc, Mineola, NY, 2000 .

[2] T. J. R. Hughes, Generalization of selective integration procedures to anisotropic and non-linear media, International Journal for Numerical Methods in Engineering 15 (9) (1980) 1413-1418.

[3] D. S. Malkus, T. J. R. Hughes, Mixed finite element methods - reduced and selective integration techniques: a unification of concepts, Computer Methods in Applied Mechanics and Engineering 15 (1) (1978) 63-81.

[4] J. C. Simo, S. Rifai, A class of mixed assumed strain methods and the method of incompatible modes, International Journal for Numerical Methods in Engineering 29 (8) (1990) 1595-1638.

[5] B. Nayroles, G. Touzot, P. Villon, Generalizing the finite element method: Diffuse approximation and diffuse elements, Computational Mechanics 10 (1992) 307-318.

[6] T. Belytschko, Y. Y. Lu, L. Gu, Element-free Galerkin methods, International Journal for Numerical Methods in Engineering 37 (2) (1994) 229-256. 
[7] W. K. Liu, S. Jun, Y. F. Zhang, Reproducing kernel particle methods, International Journal for Numerical Methods in Engineering 20 (8-9) (1995) 1081-1106.

[8] T. J. Liszka, C. A. Duarte, W. W. Tworzydlo, hp-meshless cloud method, Computer Methods in Applied Mechanics and Engineering 139 (1-4) (1996) 263-288.

[9] S. N. Atluri, T. Zhu, A new meshless local Petrov-Galerkin (MLPG) approach in computational mechanics, Computational Mechanics 22 (2) (1998) 117-127.

[10] N. Sukumar, B. Moran, T. Belytschko, The natural element method in solid mechanics, International Journal for Numerical Methods in Engineering 43 (5) (1998) 839-887.

[11] N. Sukumar, B. Moran, A. Y. Semenov, V. V. Belikov, Natural neighbour Galerkin methods, International Journal for Numerical Methods in Engineering 50 (1) (2001) 1-27.

[12] S. De, K. J. Bathe, The method of finite spheres, Computational Mechanics 25 (4) (2000) 329-345.

[13] M. Arroyo, M. Ortiz, Local maximum-entropy approximation schemes: a seamless bridge between finite elements and meshfree methods, International Journal for Numerical Methods in Engineering 65 (13) (2006) $2167-2202$.

[14] A. Huerta, S. Fernández-Méndez, Locking in the incompressible limit for 
the element-free Galerkin method, International Journal for Numerical Methods in Engineering 51 (11) (2001) 1361-1383.

[15] J. Dolbow, T. Belytschko, Volumetric locking in the element free Galerkin method, International Journal for Numerical Methods in Engineering 46 (6) (1999) 925-942.

[16] D. González, E. Cueto, M. Doblaré, Volumetric locking in natural neighbour Galerkin methods, International Journal for Numerical Methods in Engineering 61 (4) (2004) 611-632.

[17] Y. Vidal, P. Villon, A. Huerta, Locking in the incompressible limit: pseudo-divergence-free element free Galerkin, Communications in $\mathrm{Nu}-$ merical Methods in Engineering 19 (9) (2003) 725-735.

[18] D. P. Recio, R. M. N. Jorge, L. M. S. Dinis, Locking and hourglass phenomena in an element-free Galerkin context: the B-bar method with stabilization and an enhanced strain method, International Journal for Numerical Methods in Engineering 68 (13) (2006) 1329-1357.

[19] S. Beissel, T. Belytschko, Nodal integration of the element-free Galerkin method, Computer Methods in Applied Mechanics and Engineering 139 (1) (1996) 49-74.

[20] J. S. Chen, C. T. Wu, S. Yoon, Y. You, A stabilized conforming nodal integration for Galerkin mesh-free methods, International Journal for Numerical Methods in Engineering 50 (2) (2001) 435-466.

[21] J. S. Chen, W. Hu, M. Puso, Orbital HP-clouds for solving Schrödinger 
equation in quantum mechanics, Computer Methods in Applied Mechanics and Engineering 196 (37-40) (2007) 3693-3705.

[22] M. A. Puso, J. S. Chen, E. Zywicz, W. Elmer, Meshfree and finite element nodal integration methods, International Journal for Numerical Methods in Engineering 74 (3) (2008) 416-446.

[23] L. Kucherov, E. B. Tadmor, R. E. Miller, Umbrella spherical integration: a stable meshless method for non-linear solids, International Journal for Numerical Methods in Engineering 69 (13) (2007) 2807-2847.

[24] T. Belytschko, Y. Guo, W. K. Liu, S. P. Xiao, A unified stability analysis of meshless particle methods, International Journal for Numerical Methods in Engineering 48 (9) (2000) 1359-1400.

[25] T. Belytschko, S. Xiao, Stability analysis of particle methods with corrected derivatives, Computers and Mathematics with Applications $43(3-5)$ (2002) 329-350.

[26] T. P. Fries, T. Belytschko, Convergence and stabilization of stress-point integration in mesh-free and particle methods, International Journal for Numerical Methods in Engineering 74 (7) (2008) 1067-1087.

[27] J. Dolbow, T. Belytschko, Numerical integration of Galerkin weak form in meshfree methods, Computational Mechanics 23 (3) (1999) 219-230.

[28] M. Griebel, M. A. Schweitzer, A particle-partition of unity method. Part II: Efficient cover construction and reliable integration, SIAM Journal on Scientific Computing 23 (5) (2002) 1655-1682. 
[29] T. Gerstner, M. Griebel, Numerical integration using sparse grids, Numerical Algorithms 18 (3-4) (1998) 209-232.

[30] C. Riker, S. M. Holzer, The mixed-cell-complex partition-of-unity method, Computer Methods in Applied Mechanics and Engineering 198 (13-14) (2009) 1235-1248.

[31] S. N. Atluri, H. G. Kim, J. Y. Cho, A critical assessment of the truly meshless Local Petrov-Galerkin (MLPG), and Local Boundary Integral Equation (LBIE) methods, Computational Mechanics 24 (5) (1999) 348372.

[32] S. De, K. J. Bathe, The method of finite spheres with improved numerical integration, Computers and Structures 79 (22-25) (2001) 2183-2196.

[33] M. Duflot, H. Nguyen-Dang, A truly meshless Galerkin method based on a moving least squares quadrature, Communications in Numerical Methods in Engineering 18 (6) (2002) 441-449.

[34] W. Zou, J. X. Zhou, Z. Q. Zhang, Q. Li, A truly meshless method based on partition of unity quadrature for shape optimization of continua, Computational Mechanics 39 (4) (2007) 357-365.

[35] Q.-H. Zeng, D.-T. Lu, Galerkin meshless methods based on partition of unity quadrature, Applied Mathematics and Mechanics 26 (7) (2005) 893-899.

[36] A. Carpinteri, G. Ferro, G. Ventura, The partition of unity quadrature in meshless methods, International Journal for Numerical Methods in Engineering 54 (7) (2002) 987-1006. 
[37] G. R. Balachandran, A. Rajagopal, S. M. Sivakumar, Mesh free Galerkin method based on natural neighbors and conformal mapping, Computational Mechanics 42 (6) (2008) 885-905.

[38] G. R. Liu, Z. H. Tu, An adaptive procedure based on background cells for meshless methods, Computer Methods in Applied Mechanics and Engineering 191 (1) (2002) 1923-1943.

[39] I. Babuška, U. Banerjee, J. E. Osborn, Q. L. Li, Quadrature for meshless methods, International Journal for Numerical Methods in Engineering 76 (9) (2008) 1434-1470.

[40] P. Schembri, D. L. Crane, J. N. Reddy, A three-dimensional computational procedure for reproducing meshless methods and the finite element method, International Journal for Numerical Methods in Engineering 61 (6) (2004) 896-927.

[41] J. Bonet, A. J. Burton, A simple average nodal pressure tetrahedral element for incompressible and nearly incompressible dynamic explicit applications, Communications in Numerical Methods in Engineering 14 (5) (1998) 437-449.

[42] C. R. Dohrmann, M. W. Heinstein, J. Jung, S. W. Key, W. R. Witkowski, Node-based uniform strain elements for three-node triangular and four-node tetrahedral meshes, International Journal for Numerical Methods in Engineering 47 (9) (2000) 1549-1568.

[43] R. L. Taylor, A mixed-enhanced formulation for tetrahedral finite el- 
ements, International Journal for Numerical Methods in Engineering $47(1-3)(2000)$ 205-227.

[44] J. Bonet, M. Marriot, O. Hassan, An averaged nodal deformation gradient linear tetrahedral element for large strain explicit dynamic applications, Communications in Numerical Methods in Engineering 17 (8) (2001) 551-561.

[45] F. M. Andrade Pires, E. A. de Souza Neto, J. L. de la Cuesta Padilla, An assessment of the average nodal volume formulation for the analysis of nearly incompressible solids under finite strains, Communications in Numerical Methods in Engineering 20 (7) (2004) 569-583.

[46] M. A. Puso, J. Solberg, A stabilized nodally integrated tetrahedral, International Journal for Numerical Methods in Engineering 67 (6) (2006) 841-867.

[47] G. Irving, C. Schroeder, R. Fedkiw, Volume conserving finite element simulations of deformable models, ACM Transactions on Graphics 26 (3) (2007) 13.1-13.6.

[48] P. Krysl, B. Zhu, Locking-free continuum displacement finite elements with nodal integration, International Journal for Numerical Methods in Engineering 76 (7) (2008) 1020-1043.

[49] J. C. Simo, T. J. R. Hughes, On the variational foundations of assumed strain methods, Journal of Applied Mechanics 53 (1) (1986) 51-54.

[50] N. Sukumar, R. W. Wright, Overview and construction of meshfree basis functions: from moving least squares to entropy approximants, In- 
ternational Journal for Numerical Methods in Engineering 70 (2) (2007) 181-205.

[51] C. E. Shannon, A mathematical theory of communication, The Bell Systems Technical Journal 27 (1948) 379-423.

[52] E. T. Jaynes, Information theory and statistical mechanics, Physical Review 106 (4) (1957) 620-630.

[53] N. Sukumar, Construction of polygonal interpolants: a maximum entropy approach, International Journal for Numerical Methods in Engineering 61 (12) (2004) 2159-2181.

[54] J. E. Shore, R. W. Johnson, Axiomatic derivation of the principle of maximum entropy and the principle of minimum cross-entropy, IEEE Transactions on Information Theory 26 (1) (1980) 26-36.

[55] S. Li, W. K. Liu, Meshfree and particle methods and their applications, Applied Mechanics Reviews 55 (1) (2002) 1-34.

[56] T. P. Fries, H. G. Matthies, Classification and overview of meshfree methods, Tech. Rep. Informatikbericht-Nr. 2003-03, Institute of Scientific Computing, Technical University Braunschweig, Braunschweig, Germany (2004).

[57] S. Fernández-Méndez, A. Huerta, Imposing essential boundary conditions in mesh-free methods, Computer Methods in Applied Mechanics and Engineering 193 (12-14) (2004) 1257-1275. 
[58] L. L. Yaw, N. Sukumar, S. K. Kunnath, Meshfree co-rotational formulation for two-dimensional continua, International Journal for Numerical Methods in Engineering 79 (8) (2009) 979-1003.

[59] C. J. Cyron, M. Arroyo, M. Ortiz, Smooth, second order, nonnegative meshfree approximants selected by maximum entropy, International Journal for Numerical Methods in Engineering (2009) DOI: 10.1002/nme.2597.

[60] K. B. Nakshatrala, A. Masud, K. D. Hjelmstad, On finite element formulations for nearly incompressible linear elasticity, Computational Mechanics 41 (4) (2008) 547-561.

[61] J. Yvonnet, P. Villon, F. Chinesta, Natural element approximations involving bubbles for treating mechanical models in incompressible media, International Journal for Numerical Methods in Engineering 66 (7) (2006) 1125-1152.

[62] N. Sukumar, E. A. Malsch, Recent advances in the construction of polygonal finite element interpolants, Archives of Computational Methods in Engineering 13 (1) (2006) 129-163.

[63] S. P. Timoshenko, J. N. Goodier, Theory of Elasticity, 3rd Edition, McGraw-Hill, NY, 1970.

[64] D. N. Arnold, F. Brezzi, M. Fortin, A stable finite element for the Stokes equations, Calcolo 21 (4) (1984) 337-344.

[65] E. A. de Souza Neto, F. M. Andrade Pires, D. R. J. Owen, F-bar-based 
linear triangles and tetrahedra for finite strain analysis of nearly incompressible solids. Part I: formulation and benchmarking, International Journal for Numerical Methods in Engineering 62 (3) (2005) 353-383.

[66] P. Hauret, E. Kuhl, M. Ortiz, Diamond elements: a finite element/discrete-mechanics approximation scheme with guaranteed optimal convergence in incompressible elasticity, International Journal for Numerical Methods in Engineering 72 (3) (2007) 253-294.

[67] N. Kikuchi, Remarks on 4CST-elements for incompressible materials, Computer Methods in Applied Mechanics and Engineering 37 (1) (1983) 109-123. 